\title{
Beckwith Wiedemann Syndrome: A Case Report
}

\author{
K Hassan ${ }^{1}, \mathrm{AC} \mathrm{Paul}^{2}$, MR Shibli
}

\begin{abstract}
:
The baby with Beckwith Wiedemann Syndrome (BWS) usualy presents with exomphalos, macroglossia and gigantism. In addition, some children with BWS have other findings including: nevus flammeus, prominent occiput, midface hypoplasia, hemihypertrophy, genitourinary anomalies (enlarged kidneys), cardiac anomalies, musculoskeletal abnormalities, and hearing loss. Incidence of one in 13,700 live birth. As children with BWS are at increased risk of childhood cancer, they should follow up strictly for cancer screening. We are reporting this type of a case who has got Exomphalos, Macroglossia, Gigantism and Hemihypertrophy. We have corrected the exomphalos and advised him for follow up for cancer screening.
\end{abstract}

Key words: Beckwith Wiedemann Syndrome.

\section{Introduction:}

Beckwith Wiedemann Syndrome (BWS) has an estimated incidence of one in 13,700; about 300 children with BWS are born each year in the United States ${ }^{1}$. The exact incidence of BWS is unknown in our country because of the marked variability in the syndrome's presentation and difficulties with diagnosis. The number of reported infants born with BWS is most likely low because many are born with BWS, but have clinical features that are less prominent and therefore missed. BWS has been documented in a variety of ethnic groups and occurs equally in males and females.

Children conceived through In vitro fertilization have a three to four fold increased chance of developing BWS. It is thought that this is due to genes being turned on or off by the IVF procedures ${ }^{2,3}$.

The baby with BWS usualy presents with the combination of congenital abdominal wall defects as hernia (exomphalos), large tongues (macroglossia), and large bodies and/or long limbs (gigantism). In addition, some children with BWS have other findings including: nevus flammeus, prominent occiput, midface hypoplasia, hemihypertrophy, genitourinary anomalies (enlarged kidneys), cardiac anomalies, musculoskeletal abnormalities, and hearing loss. Also, some premature newborns with BWS do not have macroglossia until closer to their anticipated delivery date ${ }^{4}$.

1. Dr. Md. Kamrul Hassan, MBBS, MS (Pediatric Surgery), Associate Professor and Head, Department of Pediatric Surgery, Faridpur Medical College, Faridpur.

2. Dr Amal Chandra Paul, MBBS, MS (Pediatric Surgery), Associate Professor, Department of Pediatric Surgery, Faridpur Medical College, Faridpur.

3. Dr. Md. Mizanur Rahaman Shibli, MBBS, MS (Pediatric Surgery), Assistant Professor, Department of Pediatric Surgery, Faridpur Medical College, Faridpur.

Address of correspondence :

Dr. Md. Kamrul Hassan, MBBS, MS (Pediatric Surgery), Associate Professor and Head, Department of Pediatric Surgery, Faridpur Medical College, Faridpur. Phone: +88-01711 440 443,

Email:khassanb17@gmail.com
Another definition presented by Elliot et al. includes the presence of either three major features (anterior abdominal wall defect, macroglossia, or prepostnatal overgrowth) or two major plus three minor findings (ear pits, nevus flammeus, neonatal hypoglycemia, nephromegaly, or hemihyperplasia) $)^{5}$.

While most children with BWS do not develop cancer, children with BWS do have a significantly increased risk of cancer. Children with BWS are most at risk during early childhood and should receive cancer screening during this time ${ }^{6}$.

\section{Case Report:}

A 9-month old female patient weighing $15 \mathrm{~kg}$ was admitted in Pediatric Surgery ward of Faridpur Medical College with the complaints of a swelling over the umbilical region since birth measuring about $6 \times 5 \mathrm{~cm}$ which is reducible in lying condition. And the swelling is increased on crying. A large protruded tongue since birth. For these reasons breast feeding nor bottle feeding was possible. Right upper limb and lower limbs are larger than left one both in length \& mid thigh circumference (Fig.1).

Examination revealed that there was ventral hernia at the umbilical region. Abdomen is soft and non tender. There is a soft swelling over the umbilical region which is reducible. On musculoskeletal examination her right upper limb and lower limbs are larger than those of left. Other systemic examination reveals no apparent abnormality.

\section{Discussion:}

Abdominal wall defects are common in newborns with BWS and may require surgical treatment. Newborns with an omphalocele typically require surgery to place the abdominal contents back into the abdomen in order to prevent serious infection or shock. 


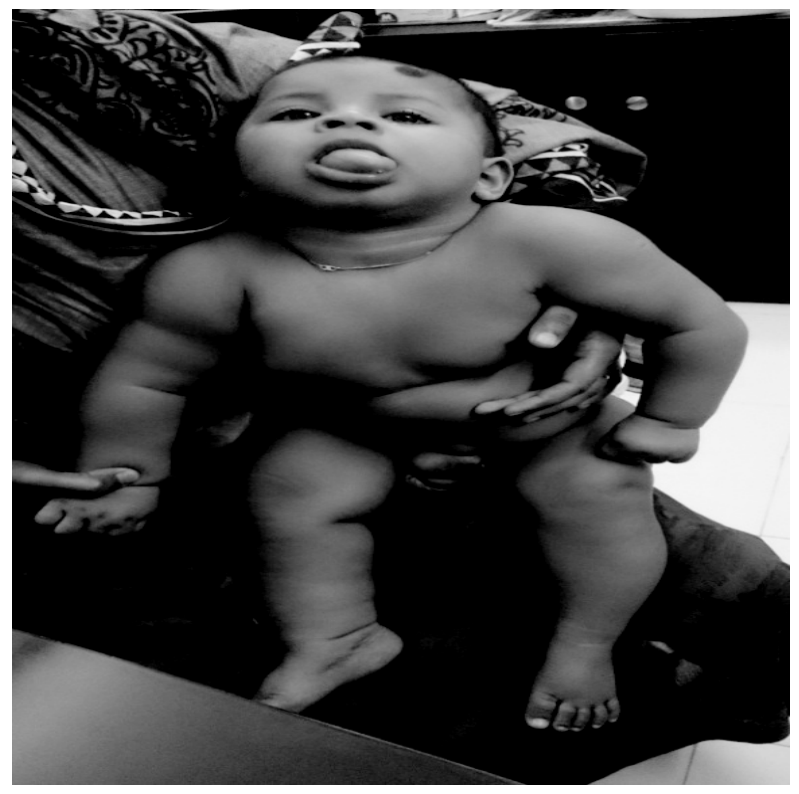

Fig-1: Exomphalos, Macroglossia and Hemihypertrophy are present in this patient

Neonatal hypoglycemia, low blood glucose in the first month of life, occurs in about half of children with BWS. ${ }^{7}$ Most of these hypoglycemic newborns are asymptomatic and have a normal blood glucose level within days. However, untreated persistent hypoglycemia can lead to permanent brain damage. Hypoglycemia in newborns with BWS should be managed according to standard protocols for treating neonatal hypoglycemia. Usually this hypoglycemia can easily be treated with more frequent feedings or medical doses of glucose. Macroglossia, a large tongue, is a very common $(>90 \%)$ and prominent feature of $\mathrm{BWS}^{8}$. Infants with BWS and macroglossia typically cannot fully close their mouth in front of their large tongue, causing it to protrude out. Macroglossia in BWS becomes less noticeable with age and often requires no treatment; but it does cause problems for some children with BWS. In severe cases, macroglossia can cause respiratory, feeding, and speech difficulties. The best time to perform surgery for a large tongue is not known. Some surgeons recommend performing the surgery between 3 and 6 months of age. Surgery for macroglossia involves removing a small part of the tongue so that it fits within the mouth to allow for proper jaw and tooth development ${ }^{5,9}$.

Hemihypertrophy (hemihyperplasia) is an abnormal asymmetry between the left and right sides of the body occurring when one part of the body grows faster than normal. Isolated hemihypertrophy is associated with a higher risk for cancer ${ }^{6,7}$. As a result, children with hemihypertrophy should follow the general cancer screening protocol for BWS. Hemihypertrophy can also cause various orthopedic problems, so children with significant limb hemihyperplasia should be evaluated and followed by an orthopedic surgeon.
Most children (>80\%) with BWS do not develop cancer; however, children with BWS are much more likely (600 times more) than other children to develop certain childhood cancers, particularly Wilms' tumor, pancreatoblastoma and hepatoblastoma, neuroblastoma, and rhabdomyosarcoma. Early diagnosis allows physicians to treat the cancer when it is at an early stage. In addition, there is less toxic treatment ${ }^{8,9}$. Given the importance of early diagnosis, all children with BWS should receive cancer screening.

\section{Conclusion:}

Children with BWS usually do very well and grow up to become the heights expected based on their parents' heights. As children with BWS are at increased risk of childhood cancer, they should follow up strictly for cancer screening. An abdominal ultrasound every 3 months until at least eight years of age is recommended and a blood test to measure alpha-fetoprotein (AFP) every 6 weeks until at least four years of age. Families and physicians should determine screening schedules for specific patients, especially the age at which to discontinue screening, based upon their own evaluation of the risk-benefit ratio.

\section{References :}

1. Elliott M, Bayly R, Cole T, Temple IK, Maher ER. "Clinical features and natural history of Beckwith-Wiedemann syndrome: presentation of 74 new cases". Clinical genetics1994; 46 (2): 168174.

2. DeBaun MR, Tucker MA "Risk of cancer during the first four years of life in children from The Beckwith-Wiedemann Syndrome Registry". The Journal of pediatrics 1998; 132 (3 Pt 1): 398-400.

3. Thorburn MJ, Wright ES, Miller CG, Smith-Read EH "Exomphalos-macroglossia-gigantism syndrome in Jamaican infants". American journal of diseases of children 1970; 119 (4): 316-21.

4. Motokura T, Bloom T, Kim HG; et al. "A novel cyclin encoded by a bcl1-linked candidate oncogene". Nature 1991; 350 (6318): 512-18.

5. DeBaun MR, Niemitz EL, McNeil DE, Brandenburg SA, Lee MP, Feinberg AP. "Epigenetic alterations of H19 and LIT1 distinguish patients with Beckwith-Wiedemann syndrome with cancer and birth defects". American Journal of Human Genetics 2002; 70 (3): 604-11.

6. Chang AS, Moley KH, Wangler M, Feinberg AP, Debaun MR. "Association between Beckwith-Wiedemann syndrome and assisted reproductive technology: a case series of 19 patients". Fertility and Sterility 2005; 83 (2): 349-354.

7. Choyke PL, Siegel MJ, Craft AW, Green DM, DeBaun MR. "Screening for Wilms tumor in children with BeckwithWiedemann syndrome or idiopathic hemihypertrophy". Medical and pediatric oncology 1999; 32 (3): 196-200.

8. Maher ER, Brueton LA, Bowdin SC. "Beckwith-Wiedemann syndrome and assisted reproduction technology (ART)". Journal of medical genetics 2003; 40 (1): 62-64.

9. Pettenati MJ, Haines JL, Higgins RR, Wappner RS, Palmer CG, Weaver DD. "Wiedemann-Beckwith syndrome: presentation of clinical and cytogenetic data on 22 new cases and review of the literature". Human Genetics 1986; 74 (2): 143-154. 